\title{
Transparencia y reputación como actitud y forma de ser de la empresa
}

\author{
Juan José Barrera Cerezal
}

\section{RESUMEN}

La transparencia en la gestión de las empresas y otro tipo de entidades privadas y públicas es un elemento central de la credibilidad y reputación frente a sus grupos de interés, además de ser un valor fundamental que caracteriza a las empresas socialmente responsables y con un buen gobierno corporativo.

La crisis económica actual ha puesto al descubierto la falta de una gestión transparente y socialmente responsable por parte de importantes empresas, dañando los intereses de accionistas, trabajadores, consumidores y su entorno social local.

La recuperación de la credibilidad de las empresas y otras entidades pasa por dar una respuesta favorable a la demanda social de mayor transparencia en la gestión, y en ese sentido los diversos acuerdos y normativas internacionales y nacionales reclaman a las empresas la elaboración de informes, que incluyan los estados financieros y, también, las actuaciones no financieras, vinculadas a ámbitos sociales y ambientales.

PALABRAS CLAVE: Transparencia, Reputación, Responsabilidad Social, Gobierno Corporativo, Cooperativa.

Cómo citar este artículo / How to cite this article: BARRERA CEREZAL, J.J. (2016): "Transparencia y reputación como actitud y forma de ser de la empresa", CIRIEC-España, Revista de Economía Pública, Social y Cooperativa, 87, 327-350.

Correspondencia: Director de Responsabilidad Social Empresarial de Gredos San Diego, S. Coop. Mad. Profesor de Formación Profesional en el ciclo formativo de Administración y Finanzas en dicha entidad. Licenciado en Ciencias Económicas y Empresariales por la Universidad Complutense de Madrid. E-mail: jj.barrera@gsd.coop. 


\section{1.- Introducción}

Hablar de transparencia y reputación de la empresa son dos conceptos estrechamente vinculados, que cada vez tienen mayor importancia en la actividad económica y social. La transparencia favorece a las empresas que creen y aplican esta forma de actuar, porque mejoran su imagen sobre los distintos actores que intervienen en el entorno de la empresa (accionistas, clientes, trabajadores, proveedores, Administraciones públicas y otros colectivos), dando respuesta clara a sus demandas, lo que hace que sea un valor destacable en su actividad.

Por el contrario, la falta de transparencia supone un elemento negativo de credibilidad para aquellas empresas que adoptan esta actitud. No se puede olvidar que una de las causas de la presente crisis económica tiene que ver con el bajo nivel de transparencia que han tenido ciertas grandes empresas, en muchos casos inexistente, cuando no engañoso, y que supone un comportamiento contrario a los principios que caracterizan un buen gobierno corporativo de las empresas.

En este artículo se pretende analizar el concepto de transparencia y el papel que tiene para configurar la reputación como actitud y forma de ser de la empresa, estrechamente relacionado con el buen gobierno corporativo de las empresas. Es evidente, que cuando se habla de transparencia debemos no solo referirnos a la empresa, sino también a otro tipo de entidades privadas y públicas, pero en este artículo se hará especial mención a la transparencia en las empresas.

\section{2.- La transparencia una necesidad irrenunciable}

En la gobernanza de las empresas es un requisito fundamental la información y el conocimiento real de la situación económico-financiera de las mismas, de hecho existe la obligación de informar a los socios y a la sociedad en general'.

1.- El Real Decreto Legislativo 1/2010, de 2 de julio, por el que se aprueba el texto refundido de la Ley de Sociedades de Capital, recoge diversas menciones a la obligación de informar por parte de las empresas, de las que cabe destacar: el artículo 93, derechos del socio, en su letra d) que recoge el derecho a la información, la Sección 2 Derecho a la Información y el Capítulo IX sobre la información societaria. 
Los casos de falta de transparencia o de información amañada han producido situaciones graves de crisis en empresas con negativos efectos en inversores, que en su gran mayoría son pequeños ahorradores, lo que pone en cuestión el propio modelo económico del mal llamado "capitalismo popular", como es el caso de las crisis producidas en el entidades financieras que afectan al conjunto del sistema financiero y que se extienden a la economía productiva.

Si el caso de Lehman Brothers es la espita que inició en el año 2007 la actual crisis financiera y después económica que aún padecemos, la falta de transparencia de estas empresas en los productos financieros que vendía y la falta de control de los reguladores públicos, mostró que el libre mercado no funciona si no hay unas reglas de juego que eviten el fraude y la falta de información clara y veraz sobre lo que se ofrece, y para que esas reglas de juego se puedan aplicar es necesario que existan árbitros que lo controlen, y esos son los reguladores, tanto en el mercado de valores como en la producción de bienes y servicios. La falta de una mayor regulación y de reguladores se echaba en falta en aquellos años de bonanza económica, década anterior a la crisis, en la que se consideraba erróneamente, como se ha demostrado, que la economía del futuro iba a avanzar por la senda del crecimiento estable, y para ello había que dejar a los mercados que fueran libres y que se autorregularan, sin interferencia de los poderes públicos.

En España, también se han producido casos muy significativos de esas malas prácticas de falta de transparencia que han dañado la credibilidad de nuestro crecimiento economía. El famoso "milagro español" se sustentaba en una financiación de tipos de interés bajos como consecuencia de la creación del euro, con un Banco Central Europeo que fijaba la política financiera de los países incorporados al Sistema Monetario Europeo, y por lo tanto de tipos de interés. La combinación de tipos de interés bajos y una tasa de inflación más alta que el resto de los países de la Unión Europea, hacia atractiva la inversión en actividades especulativas como llegó a ser el sector inmobiliario, creando una burbuja inmobiliaria, que explotó una vez que a escala internacional se entró en crisis.

El sistema financiero español pasó de ser considerado como eficaz y rentable a descubrir que su mala gestión hacía necesaria una reestructuración, y esta se ha hecho con la consecuencia de llevarse por medio la tradicional gestión social de las Cajas de Ahorros, que en su gran mayoría se han reagrupado y pasado a ser bancos, eliminando de su gestión la participación de los poderes públicos, y saneando sus cuentas a costa de endeudarse con cargo a recursos de la Unión Europea, que al final deberán pagar los ciudadanos españoles.

Entre distintos factores a tener en cuenta en esta crisis, se debe hacer mención a la opacidad y falta de transparencia de la situación real del sistema financiero español, no exento de actuaciones que pueden llegar a ser sancionadas como gestión irregular de los administradores de ciertas entidades financieras, pudiendo llegar a ser delictivas, según se puede ver en los procedimientos judiciales abiertos. 
En el periodo anterior a la crisis se hacía un discurso manipulando conceptos, se utilizada la Responsabilidad Social Empresarial (RSE) o Corporativa como cuartada para decir que no eran necesarias regulaciones públicas, y que las empresas mediante su responsabilidad corporativa podían autorregularse, obviando que la RSE no es incompatible con la existencia de normas legales que regulen la actuación de los mercados y las empresas, sino que al contrario, partiendo del cumplimiento de las normas legales existentes, la RSE favorece una mejor gobernanza de las empresas con los actores sociales².

La RSE, por lo tanto, es una apuesta de las empresas y otras entidades sociales por superar las normas legales, dando mayor valor en su actuación, en relación a conseguir un buen gobierno corporativo y una gestión con sus partes interesadas, que favorezcan una mayor competitividad y satisfacción en lo social hacia sus accionistas, clientes, trabajadores, proveedores y otros actores de su entorno.

A partir de la quiebra de Lehman Brothers, cambia la opinión pública, política y hasta de las propias empresas que desconfían de las relaciones entre sí, y se busca una mayor supervisión y una mayor regulación, y sobre todo transparencia.

Tanto en los ámbitos internacionales como los nacionales, en los últimos años se han venido produciendo diversas modificaciones normativas sobre la regulación del funcionamiento de las empresas y de los mercados que, entre otros aspectos, han ido incorporando mayor nivel de transparencia al funcionamiento de las empresas.

\section{3.- El ámbito internacional de la RSE y su incidencia en la transparencia}

Existe una estrecha relación entre el concepto de transparencia de las empresas y el de RSE. Es incongruente reconocer que una empresa se gestiona de forma socialmente responsable pero que no informa de dicha gestión o lo hace de manera opaca o no transparente.

2.- El Foro de Expertos de RSE creado por el Ministerio de Trabajo del Gobierno de España en el año 2005 definía la RSE como: "La Responsabilidad Social de la Empresa es, además del cumplimiento estricto de las obligaciones legales vigentes, la integración voluntaria en su gobierno y gestión, en su estrategia, políticas y procedimientos, de las preocupaciones sociales, laborales, medioambientales y de respeto a los derechos humanos que surgen de la relación y el diálogo transparentes con sus grupos de interés, responsabilizándose así de las consecuencias y los impactos que se derivan de sus acciones". Definición recogida, también, por el Gobierno Español en la Estrategia Española de RSE aprobada en octubre de 2014. 
Una gran parte de las referencias sobre la transparencia las encontramos en el ámbito de la RSE, por lo que en este apartado se analizará cuáles son las posiciones más destacadas que se tienen sobre el concepto de RSE en la esfera internacional.

De partida, se debe tener en cuenta que la RSE es un concepto que se enmarca en otro más amplio, el de desarrollo sostenible impulsado en los últimos años desde distintos organismos internacionales tales como Naciones Unidas, Organización Internacional del Trabajo (OIT), Organización de Cooperación y Desarrollo Económico (OCDE) y desde la Unión Europea.

De forma resumida pasemos a mencionar que elementos destaca en materia de RSE cada una de estas entidades, por cuanto son un referente a nivel internacional para los distintos actores económicos y sociales: poderes públicos, organizaciones empresarios, sindicatos de trabajadores y otras organizaciones sociales

\section{Pacto Mundial de Naciones Unidas}

Las Naciones Unidas con el objetivo de fomentar la ciudadanía corporativa propone la iniciativa internacional del Pacto Mundial que tiene como objetivo involucrar a las empresas en la gestión de algunos de los principales retos sociales y medio ambientales, consecuencia de la creciente globalización.

El Pacto Mundial establece que las empresas deben dar respuesta satisfactoria a los "Diez Principios del Pacto Mundial" que, basados en Declaraciones y Convenciones Universales, se contemplan en cuatro áreas:

- Derechos Humanos.

Principio 1: Las Empresas deben apoyar y respetar la protección de los derechos humanos fundamentales reconocidos universalmente, dentro de su ámbito de influencia.

Principio 2: Las Empresas deben asegurarse de que sus empresas no son cómplices de la vulneración de los derechos humanos

- Estándares Laborales.

Principio 3: Las empresas deben apoyar la libertad de Asociación y el reconocimiento efectivo del derecho a la negociación colectiva.

Principio 4: Las Empresas deben apoyar la eliminación de toda forma de trabajo forzoso o realizado bajo coacción.

Principio 5: Las Empresas deben apoyar la erradicación del trabajo infantil.

Principio 6: Las Empresas deben apoyar la abolición de las prácticas de discriminación en el empleo y ocupación. 
- Medio Ambiente.

Principio 7: Las Empresas deberán mantener un enfoque preventivo que favorezca el medio ambiente.

Principio 8: Las empresas deben fomentar las iniciativas que promuevan una mayor responsabilidad ambiental.

Principio 9: Las Empresas deben favorecer el desarrollo y la difusión de las tecnologías respetuosas con el medio ambiente.

- Anticorrupción.

Principio 10: Las Empresas deben trabajar en contra de la corrupción en todas sus formas, incluidas la extorsión y el soborno.

El cumplimento de estos principios puede parecer que es sencillo cuando se hace referencia a empresas que operan en países que han aprobado la Carta de derechos humanos de Naciones Unidas, pero no necesariamente es así, ya que, en un sistema como actual de economía globalizada, las empresas actúan, también, en países en los que no se cumplen las recomendaciones de Naciones Unidas sobre derechos tan básicos como los expuestos.

Las empresas multinacionales tienen delegaciones en distintos países con distintas normas sobre derechos humanos y laborales, en unos con normativas más rigurosas para el cumplimiento de los principios a los que se hace referencia, en otros países, hay una mayor laxitud para cumplirlos.

Pero no solo las empresas multinacionales se encuentran con ese escenario, sino que también afecta al resto de empresas que por vía de la cadena de valor, en concreto por sus proveedores, se pueden encontrar con que estos últimos no respeten los derechos mencionados.

Una empresa debe tener en cuenta este escenario diferente en relación a las distintas regulaciones que tienen los diversos mercados en los que opera, y si se compromete con ser una empresa socialmente responsable, debe preocuparse porque su actividad económica, incluyendo los proveedores y clientes (a veces estos últimos son otras empresas), tengan un comportamiento socialmente responsable.

El Pacto Mundial de Naciones Unidas ha habilitado un espacio en su web para que aquellas empresas que quieran funcionar con criterios socialmente responsables, puedan adherirse al cumplimento de los diez principios del Pacto Mundial.

Es importante destacar, que las empresas que se adhieran al Pacto Mundial, deberán informar de qué están haciendo para cumplir con los diez principios, y no solo en el momento de la adhesión, sino que anualmente presentarán un informe de progreso $(\mathrm{COP})^{3}$. 
Las empresas adheridas al Pacto Mundial, se exponen públicamente a que cualquier grupo de interés ${ }^{4}$ pueda criticar alguna de sus actuaciones o mostrar un desacuerdo con algún aspecto del informe, pero este riesgo de transparencia debe verse en positivo, ya que a las empresas socialmente responsables les permite adoptar medidas para corregir esas actuaciones deficientes.

El ejercicio de transparencia, informando de lo que se hace en materia de derechos humanos, relaciones laborales, medioambiente y anticorrupción, no siempre es fácil de cumplir, por eso el número de empresas y otro tipo de entidades adheridas al Pacto Mundial de Naciones Unidas no es significativamente importante. Actualmente hay algo más de 12.000 empresas y otro tipo de entidades que presentan anualmente un informe de progreso, hecho que hay que poner en valor por lo que significa de compromiso con una gestión socialmente responsable y su esfuerzo de transparencia 5 .

En este aspecto, es de destacar que hay numerosas empresas y entidades del ámbito de la Economía Social que están adheridas al Pacto Mundial, cuestión esta que va en consonancia con el carácter participativo y social que tienen este tipo de empresas, y muy en particular las cooperativas, en las que los propietarios, según el caso, son los propios trabajadores, los consumidores y usuarios o los agricultores.

\section{Declaración de la OIT}

La Declaración Tripartita de Principios sobre las Empresas Multinacionales y la Política Social de la OIT, pretende propiciar que las actuaciones de las empresas multinacionales favorezcan el progreso económico y social, y minimicen posibles efectos negativos sobre la sociedad6.

En una economía globalizada, un ámbito especial que tiene en cuenta la OIT es el papel que pueden jugar las empresas multinacionales favoreciendo la transmisión de valores sociales y laborales positivos en aquellos países en los que operan, y que no se caracterizan por respetar los más elementales derechos humanos y laborales.

En este contexto, la OIT hace referencia a necesidad de que exista una información transparente sobre aspectos que puedan afectar a la negociación colectiva, normas sobre seguridad e higiene, situación económica de la empresa y otros aspectos que puedan afectar a los ámbitos laboral y social de los trabajadores.

4.- Socios, clientes, trabajadores, proveedores, organizaciones sociales y Administraciones públicas.

5.- Empresas y entidades participantes del Pacto Mundial de Naciones Unidas: https://www.unglobalcompact.org/what-isgc/participants

6.- Recomendación de la Declaración Tripartita de la OIT: "Esta Declaración tripartita de principios tiene por objeto fomentar la contribución positiva que las empresas multinacionales pueden aportar al progreso económico y social y minimizar y resolver las dificultades a que pueden dar lugar las operaciones de estas empresas, teniendo en cuenta las resoluciones de las Naciones Unidas que preconizan el establecimiento de un nuevo orden económico internacional, así como acontecimientos que se hayan producido ulteriormente en las Naciones Unidas, por ejemplo, el Pacto Mundial y los Objetivos de Desarrollo del Milenio". 


\section{Directrices de la OCDE}

En otro orden de cosas, las líneas Directrices de la Organización de Cooperación y Desarrollo Económico (OCDE) para Empresas Multinacionales (año 2000) constituyen un conjunto de recomendaciones a las empresas multinacionales en todas las áreas importantes de la ética empresarial, incluyendo empleo y relaciones laborales, derechos humanos, medio ambiente, divulgación de información, lucha contra la corrupción, intereses de los consumidores, ciencia y tecnología, competencia y tributación.

También este organismo recoge referencias expresas a la necesidad de que las empresas sean transparentes ${ }^{7}$, y este concepto está estrechamente relacionado con una mayor demandada de información veraz realizada por los distintos actores a los que afecta la actuación de las empresas. Se pide que la información sea clara y completa, es decir, clara en cuanto a transparente y veraz, y que sea completa, es decir que no solo se suministre la información contable y financiera, sino también la no financiera, social y medioambiental entre otros aspectos ${ }^{8}$.

La Líneas Directrices de la OCDE para las empresas multinacionales, dan un especial valor a los Puntos Nacionales de Contacto (PNC), que en cada país deben crearse con la supervisión de los Gobiernos respectivos, y cuya función básicamente debe ser dar a conocer las Directrices ${ }^{9}$ y responder a las consultas que puedan realizar las organizaciones empresariales y de trabajadores, organizaciones sociales, Administraciones públicas o ciudadanos interesados.

Estos PNC, consideran como un criterio fundamental de su trabajo la transparencia ${ }^{10}$, de tal forma que la transparencia en la información es un principio que debe regir el funcionamiento de los PNC, y esta información debe proceder de las empresas multinacionales que son las destinatarias de las Directrices de la OCDE.

Cuando se habla de transparencia surge el debate sobre cuál es el nivel de transparencia que se debe tener para que, dando respuesta a demandas de los distintos grupos de interés relacionados con la empresa, se evite que se produzcan efectos indeseados que perjudiquen el funcionamiento de la empresa, por ejemplo, en relación a divulgar estrategias comerciales que beneficien a sus competidores. En este aspecto, no menor, hay que encontrar un equilibrio entre transparencia y confidencialidad.

7.- En el documento titulado "Líneas Directrices de la OCDE para empresas multinacionales, revisión 2011" se recoge la siguiente recomendación "para que se pueda conocer mejor a las empresas y sus interacciones con la sociedad y el medio ambiente, es preciso que desarrollen sus actividades con transparencia y que respondan a las demandas de información de una sociedad cada vez más exigente.

8.- Las Líneas Directrices de la OCDE para empresas multinacionales recogen: "Contar con información clara y completa sobre las empresas resulta importante para una gran cantidad de usuarios, que van desde los accionistas y la comunidad financiera hasta los trabajadores, pasando por las comunidades locales, los grupos de intereses especiales, los poderes públicos y la sociedad en su conjunto".

9.- Con este término la OCDE se refiere a las líneas directrices para empresas multinacionales.

10. - Líneas Directrices: "La transparencia es un criterio importante que contribuye favorablemente a la responsabilidad del PNC y también para lograr la confianza del público en general. Así, como principio general, las actividades del PNC serán transparentes". 
El documento de Directrices de la OCDE, plantea que las PNC tomarán las medidas que correspondan para proteger aquella "información comercial sensible" de las empresas, buscando "el equilibrio entre la transparencia y la confidencialidad con el fin de generar confianza en los procedimientos de las Directrices y para promover su implementación de forma eficaz", pero los resultados, por lo general, serán transparentes.

\section{Unión Europea}

En la Unión Europea se viene considerando como un factor clave en el desarrollo del modelo social económico las practicas socialmente responsables de las empresas.

Sin entrar en un análisis exhaustivo de las actuaciones que han venido realizando los distintos órganos políticos y administrativos de la Unión Europea en materia de fomento de la RSE, pasaré a mencionar algunas referencias que pueden ser de interés por sus efectos en el fomento de la transparencia de las empresas.

No cabe duda, que la denominada Cumbre de Lisboa, de marzo de 2000, comprometió a los primeros ministros de los países de la Unión Europea en dar un gran impulso en la dirección de reforzar las políticas europeas para posibilitar que crecimiento económico, empleo y la cohesión social.

En el documento aprobado en la Cumbre, se recoge la conclusión 39, que dice: "El Consejo Europeo hace un llamamiento especial al sentido de responsabilidad de las empresas con respecto a las prácticas idóneas en relación a la formación continua, la organización del trabajo, la igualdad de oportunidades, la integración social y el desarrollo sostenible". Esta referencia explícita a la responsabilidad de las empresas en relación con aspectos fundamentales de las relaciones laborales y, también, del desarrollo sostenible, ha ido dando paso a compromisos, 0 al menos a recomendaciones, dirigidas a desarrollar el concepto de RSE.

En el mes de julio de 2001, la Comisión Europea presenta el Libro Verde sobre RSE, bajo el título "Fomentar un marco europeo para la responsabilidad social de las empresas". Con dicho Libro, la Comisión Europea pretendía abrir un gran debate con los distintos grupos de interés, para desarrollar la RSE en el marco de la Unión Europea y resto de países.

La importancia de este documento, como referencia fundamental a las distintitas actuaciones que han venido desarrollándose, merecería de un análisis más exhaustivo que no se puede hacer en este artículo, ya que se centra en la transparencia y la reputación de las empresas, pero no quiero desaprovechar la ocasión para señalar, que el Libro Verde de RSE hace una mención a las empresas de Economía Social, en el sentido de mencionar que "las cooperativas de trabajadores y los sistemas de participación, así como otras formas de empresas (cooperativas, mutualistas o asociativas) integran estructuralmente los intereses de otros interlocutores y asumen espontáneamente responsabilidades sociales y civiles". 
El Libro Verde de RSE hace una referencia expresa a la "transparencia" y a "la fiabilidad de la evaluación y la validación"11.

Estos conceptos de transparencia y fiabilidad de la evaluación y validación, unidos al concepto de la voluntariedad ${ }^{12}$ de la RSE, han sido y siguen siendo una constante en el debate sobre cuál debe ser el alcance que cada uno de estos conceptos tiene para cada uno de los grupos de interés.

La Comisión Europea está comprometida con propiciar la transparencia y la evaluación y validación, pero para llevarlo a cabo se pregunta sobre cuál puede ser su papel en la promoción de la transparencia ${ }^{13}$, y cuál es la mejor forma de evaluar distintos aspectos que incorpora la RSE ${ }^{14}$.

Las respuestas a estas preguntas se pueden ver en el desarrollo de propuestas sobre RSE que ha venido elaborando la Comisión Europea a lo largo de los últimos años, y que han nacido del debate interno en las reuniones del Grupo de Alto Nivel sobre RSE constituido por la Comisión Europea y formado por los responsables de fomento de la RSE en cada país miembro de la Unión Europea, y de las reuniones que se han tenido con otros actores europeos en materia de RSE: organizaciones empresariales y sindicales y otras organizaciones sociales (Foro multistakeholder europeo de RSE).

Del resultado de dichos foros, la Comisión ha ido aprobando diversas "Comunicaciones" en materia de RSE que eran dirigidas a distintas instancias europeas: el Parlamento, la Comisión, el Consejo Económico y Social y el Comité de las Regiones, además, de ser documentos públicos para el conocimiento de cualquier ciudadano. En dichas Comunicaciones se hacen recomendaciones y propuestas para la aprobación de medidas para que sean adoptadas por las entidades competentes, tanto del ámbito de la Unión Europea, como por cada gobierno de cada país miembro de la Unión.

11.- Referencia a la transparencia en el texto del Libro Verde de RSE de la Comisión Europea:

"El presente Libro Verde tiene por objeto iniciar un amplio debate sobre cómo podría fomentar la Unión Europea la responsabilidad social de las empresas a nivel europeo e internacional, en particular sobre cómo aprovechar al máximo las experiencias existentes, fomentar el desarrollo de prácticas innovadoras, aumentar la transparencia e incrementar la fiabilidad de la evaluación y la validación".

12.- Referencia en el Libro Verde sobre RSE:

"La responsabilidad social de las empresas es, esencialmente, un concepto con arreglo al cual las empresas deciden voluntariamente contribuir al logro de una sociedad mejor y un medio ambiente más limpio".

13.- Referencia en el Libro Verde sobre RSE:

"El papel de la UE

¿Qué podría hacer la Unión Europea para fomentar el desarrollo de la responsabilidad social de las empresas a nivel europeo e internacional? En particular, ¿debería la UE aportar un valor añadido a las actividades socialmente responsables y completarlas:

- elaborando un marco europeo general, en asociación con los principales agentes en el ámbito de la responsabilidad social de las empresas, tendente a promover la transparencia, la coherencia y las buenas prácticas en materia de responsabilidad social;

- fomentando el consenso sobre las buenas prácticas en materia de evaluación y control de la responsabilidad social de las empresas y apoyando dichas prácticas;

- o por qué otro medio?

14.- Referencia en el Libro Verde sobre RSE

Evaluación y eficacia

- ¿Cuál es la mejor manera de elaborar, evaluar y garantizar la eficacia y la fiabilidad de instrumentos de responsabilidad social empresarial tales como los códigos de conducta, los informes y auditorías sociales, las etiquetas sociales y ecológicas y la inversión socialmente responsable? 


\section{Comunicación de la Unión Europea}

En materia de RSE ha habido varias comunicaciones, pero la actualmente vigente corresponde a la Comunicación que realiza la Comisión Europea sobre RSE dirigida al Parlamento Europeo, al Consejo, al Comité Económico y Social Europeo y la Comité de las Regiones el 25 de octubre de 2011.

Bajo el título de "Estrategia renovada de la UE para 2011-2014 sobre la responsabilidad social de las empresas", la Comisión actualiza la definición de RSE, titulando "La responsabilidad de las empresas por su impacto en la sociedad" y dice que:

"Las empresas deben aplicar, en estrecha colaboración con las partes interesadas, un proceso destinado a integrar las preocupaciones sociales, medioambientales y éticas, el respeto de los derechos humanos y las preocupaciones de los consumidores en sus operaciones empresariales y su estrategia básica, a fin de:

- maximizar la creación de valor compartido para sus propietarios/accionistas y para las demás partes interesadas y la sociedad en sentido amplio

- identificar, prevenir y atenuar sus posibles consecuencias adversas".

Con esta definición, la Comisión da un paso más en llamar la atención a las empresas sobre la importancia de las prácticas de RSE como creadoras de valor que debe ser compartido entre los propietarios de las empresas, sus accionistas, y, también, con las demás partes interesadas. Y ¿quién son estas últimas?, pues fundamentalmente los consumidores, los trabajadores, los proveedores y el ámbito social que rodea a las empresas. Generar beneficios económicos que retribuyan a los inversionistas no debe ser incompatible con prestar un buen servicio a los clientes, mejorar las condiciones laborales de los zempleados, no deteriorar el medio ambiente o cuidar del entorno social local donde operan las empresas.

También, la nueva Comunicación de la Comisión hace referencia a identificar, prevenir y atenuar las posibles consecuencias adversas que pudiera tener la acción de las empresas. Este tema cada vez adquiere una mayor importancia, ya que hay prácticas empresariales que están produciendo efectos negativos indeseables, sirva como ejemplo la ligereza con que se ha tratado por las entidades financieras la financiación de la burbuja inmobiliaria, y que tan costoso está suponiendo para la totalidad de los ciudadanos financiar el saneamiento del sistema financiero, pagando no solo con impuestos sino también con la pérdida del empleo dicha irresponsabilidad, que además se agrava en el caso de algunas familias que, habiendo perdido el empleo y sin recursos económicos suficientes, no pueden hacer frente a préstamos concedidos por estas entidades, y donde ahora se aplica la normativa sobre insolvencia con una excesiva rigidez, provocando desahucios que dejan a familias en la calle. 
Teniendo en cuenta los aspectos descritos, hay que preguntarse ¿qué abarca la RSE?, y la respuesta lleva a establecer que la RSE básicamente hace referencia a:

- El respeto a los derechos humanos

- Prácticas de trabajo y de empleo (como la formación, respeto a la diversidad, la igualdad de género y la salud y el bienestar de los trabajadores), que favorezcan el entorno social donde opera la empresa.

- Las cuestiones medioambientales (como la biodiversidad, el cambio climático, el uso eficiente de los recursos, la evaluación del ciclo de vida y la prevención de la contaminación)

- La lucha contra el fraude y la corrupción

- La participación de las comunidades locales y el desarrollo

- La integración de las personas con discapacidad

- Los intereses de los consumidores, incluida la intimidad

No cabe duda que estas características de la RSE tienen mucho que ver con las empresas y entidades de la Economía Social, y en particular con las cooperativas y las mutualidades como así lo recoge la propia Comunicación de la Comisión ${ }^{15}$.

La Comunicación de la Comisión realiza diversas propuestas a los Estados de la UE que merecen ser tenidas en cuenta. En particular, y en relación al tema que tratamos sobre transparencia, la Comisión pretende fomentar la elaboración de informes de RSE y para ello quiere llevar a cabo un seguimiento del comportamiento de las grandes empresas europeas, en concreto por aquellas que tengan más de 1000 trabajadores $^{16}$.

Este aspecto es de especial relevancia, ya que en el ámbito de la RSE se viene produciendo un intenso debate sobre si debe haber algún tipo de medidas desde las instancias de la Unión Europea que obligue a las empresas a que informen sobre lo que están haciendo sobre gestión socialmente responsable.

Si bien la Comunicación de octubre de 2011 recoge indirectamente una recomendación para informar, recientemente se ha dado un nuevo avance en propiciar la información de materias no financieras de las empresas, adquiriendo una mayor obligación de informar sobre aspectos vinculados con la RSE, y que esta se haga en empresas con más de 500 trabajadores ${ }^{17}$.

15.- Algunos tipos de empresas, como las cooperativas, las mutuas y las empresas familiares, tienen estructuras de propiedad y de gobernanza que pueden ser especialmente propicias para una gestión empresarial responsable.

16.- La Comisión se propone hacer un seguimiento del compromiso adquirido por las empresas europeas con más de 1000 trabajadores de tomar en consideración los principios y directrices sobre RSE y la norma de orientación ISO 26000 sobre responsabilidad social en sus operaciones. La Comisión invita a todas las grandes empresas europeas a comprometerme de aquí a 2014 a tomar en consideración al menos uno de los siguientes conjuntos de principios y directrices al elaborar su planteamiento sobre la RSE: el Pacto Mundial de las Naciones Unidas, las Líneas Directrices de la OCDE para las empresas multinacionales o la norma guía ISO 26000 sobre responsabilidad social. 
Esta directiva es un paso cualitativo importante en el compromiso de la Unión Europea por fomentar la RSE y por la información y la transparencia, como así lo recoge esta Directiva ${ }^{18}$. Ahora les queda a los países miembros regular las normas necesarias para su cumplimiento, y para ello tienen un plazo de hasta el 6 de diciembre de 2016, y el primer ejercicio económico a tener en cuenta por las empresas en sus informes será el correspondiente al año $2017^{19}$.

\section{4.- La transparencia en la regulación empresarial en España}

Para hablar de la transparencia en el ámbito de España, ligando este concepto al de la RSE, es conveniente hacer una breve referencia a la reciente historia de la evolución de la RSE en España.

Existen diversos antecedentes, de los que se analizarán para este estudio los que se han producido desde el año 2005, ya que durante estos diez últimos años ha habido diversos hechos que tienen una especial relevancia en materia de RSE como a continuación se expondrá.

Las empresas españolas, y en concreto las empresas multinacionales con domicilio en España, se han visto en la necesidad de dar respuesta a una demanda que cada vez más se hacía patente, y es que como grandes empresas fueran en sus actuaciones transparentes y socialmente responsables. Como se ha dicho en apartados anteriores se reclamaba este comportamiento por distintos actores e instituciones internacionales como Naciones Unidas, OIT, OCDE y Unión Europea.

17.- La Directiva 2014/95/UE del Parlamento Europeo y del Consejo de 22 de octubre de 2014 establece:

Las grandes empresas que sean entidades de interés público que, en sus fechas de cierre del balance, superen el criterio de un número medio de empleados superior a 500 durante el ejercicio, incluirán en el informe de gestión un estado no financiero que contenga información, en la medida en que resulte necesaria para comprender la evolución, los resultados y la situación de la empresa, y el impacto de su actividad, relativa, como mínimo, a cuestiones medioambientales y sociales, así como relativas al personal, al respeto de los derechos humanos y a la lucha contra la corrupción y el soborno, y que incluya: a) una breve descripción del modelo de negocio de la empresa; b) una descripción de las políticas que aplica la empresa en relación con dichas cuestiones, que incluya los procedimientos de diligencia debida aplicados; c) los resultados de esas políticas; d) los principales riesgos relacionados con esas cuestiones vinculados a las actividades de la empresa, entre ellas, cuando sea pertinente y proporcionado, sus relaciones comerciales, productos o servicios que puedan tener efectos negativos en esos ámbitos, y cómo la empresa gestiona dichos riesgos; e) indicadores clave de resultados no financieros, que sean pertinentes respecto de la actividad empresarial concreta. En el caso de que la empresa no aplique ninguna política en relación con una o varias de esas cuestiones, el estado no financiero ofrecerá una explicación clara y motivada al respecto.

18.- En su Comunicación titulada «Acta del Mercado Único - Doce prioridades para estimular el crecimiento y reforzar la confianza "Juntos por un nuevo crecimiento"” adoptada el 13 de abril de 2011, la Comisión señalaba la necesidad de aumentar la transparencia de la información social y medioambiental facilitada por las empresas de todos los sectores a un nivel alto similar en todos los Estados miembros. Esto es totalmente coherente con la posibilidad de que los Estados miembros exijan, en su caso, nuevas mejoras en la transparencia de la información no financiera de las empresas, lo cual por su propia naturaleza supone un esfuerzo continuo.

19.- Los Estados miembros pondrán en vigor las disposiciones legales, reglamentarias y administrativas necesarias para dar cumplimiento a lo establecido en la presente Directiva a más tardar el 6 de diciembre de 2016. Informarán de ello inmediatamente a la Comisión. Los Estados miembros establecerán que las disposiciones contempladas en el párrafo primero se apliquen a todas las empresas incluidas en el ámbito de aplicación del artículo 1, en lo que respecta al ejercicio que comienza el 1 de enero de 2017 o durante el año civil de 2017. 
También, es importante resaltar que hay empresas españolas que operan en países desarrollados en los que se establecen normativas sobre gestión socialmente responsable, y que exigen a las empresas que informen de diversos aspectos económicos, sociales y medioambientales si quieren participar en las Bolsas de Valores de esos países, con indicadores como el Dow Jones Sustainability Index que mide, además de los estados financieros de una empresa, la gestión sobre aspectos sociales y ambientales, lo que hace necesario un compromiso con la RSE, pero también, cuando se opera en países en los que no se respetan los más elementales derechos humanos y de relaciones laborales, es necesario trasladar comportamientos de gestión de RSE, porque esas prácticas dan un valor añadido a la función de la empresa y confirman unas señas de identidad de empresa socialmente responsable que favorece su imagen corporativa en esos países.

En este escenario, las empresas españolas han venido demandando un acompañamiento de los poderes públicos para poder desarrollar una política de fomento de la RSE.

En el año 2002 el grupo parlamentario socialista presentó en el Congreso de los Diputados una Proposición de Ley que pretendía regular la RSE. Fue rechazada por la mayoría del grupo parlamentario popular en mayo de 2002, quien a su vez, el 25 de noviembre de 2002, presentó una Proposición no de Ley por la que se creaba un grupo de expertos en RSE, compuesto por 13 miembros que coordinaría la Dirección General de Economía Social y del Fondo Social Europeo.

El Gobierno socialista, que surge de las elecciones de marzo de 2004, inicia un proceso de elaboración de un marco de medidas que fomenten la RSE en España, dentro de los objetivos recogidos en la Estrategia Europea de Desarrollo Sostenible.

La intención de aquel Gobierno era que la política de fomento de la RSE naciera del diálogo con los interlocutores sociales y la sociedad civil en su conjunto, por lo que se tuvo en cuenta el resultado de tres foros:

- Subcomisión Parlamentaria

- Foro de Expertos sobre RSE creado por el Gobierno

- Mesa del diálogo social formada por el Gobierno y las organizaciones de empresarios y de trabajadores.

\section{La Subcomisión Parlamentaria}

La Subcomisión parlamentaria se creó en el Congreso de los Diputados en el año 2005, y estuvo formada por representantes de todos los grupos parlamentarios. Se solicitó la presencia de representantes de la Administración General del Estado, de empresas, organizaciones empresariales y sin- 
dicales, ONGs y expertos, y a partir de sus opiniones, la Subcomisión elaboró y aprobó un documento con 56 recomendaciones dirigidas a los poderes públicos, empresas y organizaciones sociales ${ }^{20}$.

\section{El Foro de Expertos}

El Foro de Expertos se constituyó en marzo de 2005, estuvo formado por 47 miembros, que representaban a fundaciones y asociaciones que agrupaban a empresas que promocionaban la RSE; organizaciones no gubernamentales que defienden el medio ambiente, los derechos humanos, al consumidor, la economía social, etc...; expertos procedentes del mundo académico y de la investigación sobre RSE y distintos departamentos ministeriales del Gobierno.

Su trabajo finalizó en el año 2007 con la aprobación de un documento que contemplaba 29 recomendaciones dirigidas a los distintos grupos de interés. ${ }^{21}$

\section{El Diálogo Social}

En el ámbito del Diálogo Social que se produjo en el año 2007, las organizaciones empresariales y sindicales y el Gobierno llegaron, el 19 de diciembre de 2007, a un documento de acuerdo que incluye 10 propuestas para la promoción y el desarrollo de la RSE22.

El interés de los documentos aprobados merecería de un análisis detallado de los mismos, pero no parece que sea este artículo el lugar para hacerlo, aunque si es importante destacar que son documentos que han servido de referencia para la elaboración de las distintas medidas y normativa que de forma directa o indirecta han ido configurando la política de fomento de la RSE en España, además de ser un referente que aportó ideas en los debates del Grupo de Alto Nivel sobre RSE de la Comisión Europea.

\section{El Consejo Estatal de RSE}

Dentro de todas las recomendaciones que se realizaron en los tres foros, cabe destacar la coincidencia en pedir la creación de un Órgano Administrativo que sirviera de lugar de debate y generación de propuestas de los distintos grupos de interés que están en torno a la RSE.

20.- Libro titulado "Informe del Congreso de los Diputados para potenciar y promover la responsabilidad social de las empresas" editado por el Ministerio de Trabajo y Asuntos Sociales.

21.- Libro titulado "Responsabilidad Social de las Empresas", editado por el Ministerio de Trabajo y Asuntos Sociales, año 2008, que contiene los documentos: Informe del Foro de Expertos en Responsabilidad Social de las Empresas; Informe sobre aspectos sociales y medioambientales relacionados con la Responsabilidad Social de las Empresas.

22.- Libro titulado "Responsabilidad Social de las Empresas", editado por el Ministerio de Trabajo y Asuntos Sociales, año 2008, que contiene los documentos: Informe del Foro de Expertos en Responsabilidad Social de las Empresas; Informe sobre aspectos sociales y medioambientales relacionados con la Responsabilidad Social de las Empresas. 
A pesar de la diferencia de posiciones de los distintos grupos de interés, siempre se ha dado un espíritu de diálogo sobre RSE en España, por lo que el Gobierno de aquel momento aprobó la creación del Consejo Estatal de Responsabilidad Social de las Empresas, CERSE, que nace el 15 de febrero de 2008, mediante la aprobación por el Consejo de Ministros del Real Decreto de creación del mismo, dando respuesta a las recomendaciones planteadas por el Parlamento, el Foro de Expertos de RSE y la Mesa del Diálogo Social sobre RSE.

Se crea como órgano colegiado interministerial, de carácter asesor y consultivo del Gobierno, de composición cuatripartita y paritaria (organizaciones empresariales, sindicatos, otras organizaciones sociales y Administraciones públicas), adscrito al Ministerio de Trabajo, hoy de Empleo y Seguridad Social, a través de la Secretaría de Estado de Empleo.

El CERSE está encargado del impulso y fomento de las políticas de Responsabilidad Social de las Empresas y se pretende que constituya el marco de referencia para el desarrollo de esta materia en España.

La normativa que regula el CERSE contempla ${ }^{23}$, entre una de sus funciones, constituirse como Observatorio de la RSE en España, lo que tiene una especial importancia en relación con la información y la transparencia de las empresas en materia de RSE, y en particular con la información de las empresas con más de 1000 trabajadores, que como se ha visto con anterioridad también recoge la Comisión Europea en su Comunicación de 25 de octubre de 2011, con la propia Directiva del Parlamento y la Comisión sobre informes no financieros de las empresas con más de 500 trabajadores y con lo establecido en materia de información de RSE en la Ley de Economía Sostenible aprobada en el año 2011 y que veremos a continuación.

\section{Ley de Economía Sostenible}

Una de las referencias más importantes en materia de RSE y de su incidencia en la información transparente, se hace en la Ley 2/2011, de 4 de marzo, de Economía Sostenible, que en su artículo 2 recoge una definición de economía sostenible24, que posteriormente da pie a en un Capítulo sexto que establece de forma clara cuál es la política de fomento de la RSE que deben desarrollar los poderes públicos.

23.- Libro titulado "Responsabilidad Social de las Empresas", editado por el Ministerio de Trabajo y Asuntos Sociales, año 2008, que contiene los documentos: Informe del Foro de Expertos en Responsabilidad Social de las Empresas; Informe sobre aspectos sociales y medioambientales relacionados con la Responsabilidad Social de las Empresas

24.- Ley 2/2011, de 4 de marzo, de Economía Sostenible. Artículo 2. Economía sostenible.

A los efectos de la presente Ley, se entiende por economía sostenible un patrón de crecimiento que concilie el desarrollo económico, social y ambiental en una economía productiva y competitiva, que favorezca el empleo de calidad, la igualdad de oportunidades y la cohesión social, y que garantice el respeto ambiental y el uso racional de los recursos naturales, de forma que permita satisfacer las necesidades de las generaciones presentes sin comprometer las posibilidades de las generaciones futuras para atender sus propias necesidades. 
En concreto, se establecen cuáles son los objetivos de la RSE según el legislador, en los que se pone como primer objetivo la transparencia en la gestión ${ }^{25}$, y esta debe venir acompañada de la necesaria información de las empresas, y aquí se destaca el papel de los informes de RSE, y sobre todo el que deben realizar las empresas de más de 1000 trabajadores 26 . Hay que llamar la atención, a que la Ley de Economía Sostenible se aprueba en marzo de 2011, fecha anterior a octubre de 2011 cuando la Comisión Europea aprueba su Comunicación sobre RSE que también hace una referencia similar. La representación española había expuesto en una reunión del Grupo de Alto Nivel sobre RSE de la Comisión Europea su Ley de Economía Sostenible antes de la aprobación de dicha Comunicación.

La Ley de Economía Sostenible, también, recoge en una disposición adicional el mandato al Gobierno para que reglamentariamente se desarrollen las condiciones para la difusión de la información sobre el uso de criterios sociales, medioambientales y de buen gobierno en la política de inversión de los fondos de pensiones.

Este tema es de especial importancia, ya que los fondos de pensiones manejan cuantiosos recursos económicos y, por lo tanto, sus decisiones de inversión inciden de forma muy destacada en el comportamiento de las empresas cotizadas y en la cadena de valor, sus proveedores. Sus decisiones, junto a las efectuadas por los fondos de inversión, tienen que ver con el fomento de la Inversión Socialmente Responsable.

De igual forma, también, se recoge que las sociedades mercantiles estatales adscritas a la Administración General del Estado, en el plazo de un año, desde la entrada en vigor de la Ley, adaptarán sus planes estratégicos para:

- Presentar anualmente informes de gobierno corporativo, así como memorias de sostenibilidad de acuerdo con estándares comúnmente aceptados.

- Favorecer la adopción de principios y prácticas de responsabilidad social empresarial por sus proveedores.

25.- El conjunto de características, indicadores y modelos de referencia a que se refiere el apartado anterior deberá atender especialmente a los objetivos de transparencia en la gestión, buen gobierno corporativo, compromiso con lo local y el medioambiente, respeto a los derechos humanos, mejora de las relaciones laborales, promoción de la integración de la mujer, de la igualdad efectiva entre mujeres y hombres, de la igualdad de oportunidades y accesibilidad universal de las personas con discapacidad y del consumo sostenible, todo ello de acuerdo con las recomendaciones que, en este sentido, haga el Consejo Estatal de la Responsabilidad Social Empresarial, constituido por el Real Decreto 221/2008, de 15 de febrero, por el que se regula el Consejo Estatal de Responsabilidad Social de las Empresas.

26.- Las sociedades anónimas podrán hacer públicos con carácter anual sus políticas y resultados en materia de Responsabilidad Social Empresarial a través de un informe específico basado en los objetivos, características, indicadores y estándares internacionales mencionados en los apartados anteriores. En todo caso, en dicho informe específico deberá constar si ha sido verificado o no por terceras partes.

En el caso de sociedades anónimas de más de 1.000 asalariados, este informe anual de Responsabilidad Social Empresarial será objeto de comunicación al Consejo Estatal de Responsabilidad Social Empresarial que permita efectuar un adecuado seguimiento sobre el grado de implantación de las políticas de Responsabilidad Social Empresarial en las grandes empresas españolas.

Asimismo, cualquier empresa podrá solicitar voluntariamente ser reconocida como empresa socialmente responsable, de acuerdo con las condiciones que determine el Consejo Estatal de Responsabilidad Social Empresarial. 
No cabe duda, que la Ley de Economía Sostenible ha sido un gran avance regulatorio de muchos aspectos, y en particular de la RSE, y de la necesidad de que las empresas y otro tipo de entidades informen de forma transparente de lo que hacen, y no solo sobre aspectos financieros, sino también de aspectos no financieros.

Por desgracia, transcurrido cuatro años de la aprobación de la Ley de Economía Sostenible, muchas de las medidas recogidas, y en particular las referentes a la información de las empresas en materia de RSE, no se están llevando a cabo. Al menos, se desconoce que las sociedades anónimas de más de 1000 trabajadores informen al CERSE sobre lo que hacen en materia de RSE, para que este Consejo pueda emitir un informe sobre cómo evoluciona la RSE en España; no se ha habilitado ningún procedimiento para que las empresas que voluntariamente lo soliciten puedan ser reconocidas como socialmente responsables; no se conoce que los fondos de pensiones informen sobre sus inversiones en materia de Inversión Socialmente Responsable y tampoco se conoce que se haya establecido un sistema de elaboración de informes de sostenibilidad en las empresas públicas, independientemente de que algunas de ellas realicen dichos informes.

\section{Estrategia Española sobre RSE}

En este escenario, el Gobierno actual aprobó el 24 de octubre de 2014 la Estrategia Española sobre RSE, mediante el título de "Estrategia 2014-2020 para empresas, administraciones públicas y el resto de organizaciones para avanzar hacia una sociedad y una economía más competitiva, productiva, sostenible e integradora"27.

El objetivo es constituirse como un elemento de orientación y de apoyo al desarrollo de prácticas socialmente responsables para todas las empresas, las Administraciones Públicas, y las diferentes organizaciones públicas y privadas.

La Estrategia va dirigida a promover la responsabilidad social en el mayor número de organizaciones, teniendo en cuenta 6 principios: competitividad, cohesión social, creación de valor compartido, sostenibilidad, transparencia y voluntariedad.

Como se puede ver, la transparencia sigue teniendo un valor importante en la RSE, siendo considerado como un principio estratégico. 
Para llevar a cabo el cumplimento de esos 6 principios, se establecen 10 líneas de actuación:

1. Promoción de la RSE como elemento impulsor de organizaciones más sostenibles.

2. Integración de la RSE en la educación, la formación y en la investigación.

3. Buen Gobierno y transparencia como instrumentos para el aumento de la confianza.

4. Gestión responsable de los recursos humanos y fomento del empleo.

5. Inversión socialmente responsable e l+D+i.

6. Relación con los proveedores.

7. Consumo responsable.

8. Respeto al medioambiente.

9. Cooperación al desarrollo.

10. Coordinación y participación

La tercera línea de actuación, relaciona la transparencia con el buen gobierno de las empresas y otro tipo de entidades con el objeto de aumentar la confianza, lo que está muy bien traído teniendo en cuenta los acontecimientos que se han dado en los últimos tiempos, ya que las malas prácticas que se han realizado por algunas empresas y ciertos sectores han producido una desconfianza entre las partes interesadas, y no solo en los consumidores y trabajadores, sino también en los propios accionistas.

Dentro de las 60 medidas de actuación que recogen la Estrategia Española de RSE hay algunas que merecen mención por su incidencia en la transparencia:

- Puesta en marcha del procedimiento de publicación de memorias e informes de responsabilidad social y sostenibilidad dando respuesta a lo previsto en la Ley 2/2011, de 4 de marzo, de Economía Sostenible.

- El CERSE seguirá trabajando para alcanzar una cierta homogeneidad en los estándares de reporte utilizados de las Memorias RSE.

- Se habilitará el tratamiento de las memorias de las empresas de más de 1.000 trabajadores, conforme a lo que establece la Ley 2/2011, de 4 de marzo, de Economía Sostenible, reconociendo además a las entidades que lo hagan.

- Fomentar la elaboración de informes de gobierno corporativo y memorias de sostenibilidad tanto entre las sociedades mercantiles estatales como las entidades públicas empresariales adscritas a la Administración General del Estado, siguiendo el modelo que se establezca en el seno del CERSE.

- Fomentar que las entidades gestoras de fondos de inversión y planes de pensiones, indiquen si tienen en cuenta solo criterios financieros en las decisiones de inversión y, cuando tengan en consideración riesgos extra financieros, mencionen esta circunstancia.

- Extender la utilización del etiquetado como instrumento de información al consumidor y herramienta informativa del cumplimiento de criterios de sostenibilidad. 
Como se puede comprobar, la Estrategia reitera las medidas que desde los foros del Parlamento, de Expertos y Mesa del Diálogo Social se recogían en sus recomendaciones, haciendo referencia expresa a normas como el Real decreto de 2008 sobre la creación del CERSE y la Ley de Economía Sostenible.

Sería deseable que más pronto que tarde se pueda empezar a desarrollar en concreto las medidas recogidas en dichas normas y en la propia Estrategia.

\section{Código de Buen Gobierno de la CNMV}

La aprobación del nuevo Código de Buen Gobierno de la Comisión Nacional del Mercado de Valores (CNMV), en febrero de 2015, es un gran paso en el nivel de exigencia de buenas prácticas y de transparencia de las empresas cotizadas.

El nuevo Código pretende dar respuesta a las malas prácticas empresariales, caracterizadas en la opacidad en la información y mal gobierno corporativo que se han venido produciendo durante los años del tan elogiado periodo de crecimiento económico, y que son una de las importantes causas de la actual crisis económica.

Intentar buscar más transparencia y un mejor gobierno corporativo son los objetivos que persigue el nuevo Código, como así se desprende del documento aprobado28.

En este escenario, el nuevo Código, a diferencia del anterior de 2006, hace hincapié sobre la importancia de la RSE, considerando que es un principio que el Consejo de Administración promueva una política de RSE y que informe de forma transparente sobre su desarrollo, aplicación y resultados 29 . Destaca esta obligación, ya que la RSE pasa a formar parte de la estrategia de la empresa, y al ser aprobada por el Consejo de Administración, refuerza el compromiso de toda la organización con las prácticas de gestión socialmente responsables. Es una forma de gestionar la empresa, no es una parte más de un área de actuación, sino que tiene que impregnar toda la actuación de gestión de la empresa.

En este sentido, se recomienda se cree una comisión del Conejo de Administración específica para impulsar y llevar un seguimiento de la gestión de un buen gobierno corporativo y de la RSE30.

28.- En los últimos años hemos asistido a una proliferación de iniciativas relacionadas con las buenas prácticas en materia de gobierno corporativo, cuya intensidad se ha multiplicado a partir del inicio de la crisis financiera internacional, por el convencimiento generalizado de la importancia que tiene que las sociedades cotizadas sean gestionadas de manera adecuada y transparente como factor esencial para la generación de valor en las empresas, la mejora de la eficiencia económica y el refuerzo de la confianza de los inversores.

29.- Principio 24: La sociedad promoverá una política adecuada de responsabilidad social corporativa, como facultad indelegable del consejo de administración, ofreciendo de forma transparente información suficientes obre su desarrollo, aplicación y resultados

30.- Dada la relevancia de las cuestiones relativas al gobierno corporativo y a la responsabilidad social corporativa, se recomienda la identificación y atribución de funciones específicas en esta materia a una comisión especializada, que podrá ser la comisión de auditoría, la comisión de nombramientos o una comisión ad hoc de gobierno corporativo y responsabilidad social, con el objetivo de impulsar una gestión de estos asuntos más intensa y comprometida. 
El diálogo entre los distintos grupos de interés es fundamental en el desarrollo y credibilidad de la gestión socialmente responsable de la empresa, por esa razón es básico una comunicación que permita exponer aspectos importantes de la RSE, y para ello se recomienda utilizar indicadores reconocidos internacionalmente ${ }^{31}$.

\section{5.- La transparencia como valor reputacional y buen gobierno corporativo}

Llegados a este punto, tras haber expuesto las referencias internacionales y en España de la RSE y la transparencia, parece conveniente hacer una reflexión sobre los valores que comporta la RSE para las empresas que la llevan a cabo.

Cada vez más se ve la necesidad de informar sobre lo que se hace en la empresa en materia de responsabilidad social. Cada vez hay más informes de RSE. España es uno de los países con más empresas y entidades adheridas al Pacto Mundial de Naciones Unidas, con lo que ello comporta de tener que informar anualmente de los progresos que se hacen en el cumplimiento y desarrollo de los 10 principios. Pero igual que se destacan estos aspectos, también hay que decir, que las empresas españolas tienen una posición contraria a tener que informar por requerimiento de una norma legal.

La demostración práctica de esta actitud negativa a informar por obligación, se observa cuando se manifiestan posiciones contrarias a la implantación de la Ley de Economía Sostenible en cuanto a que las empresas de más de 1000 trabajadores informen que acciones realizan en materia de RSE.

El positivo valor reputacional que tienen las empresas que se gestionan de forma socialmente responsable es reconocido por distintos estudios. Y dentro de los factores que contempla la RSE ligada a la reputación, la transparencia en la gestión es uno de los valores que más resaltan las empresas. Tomando como ejemplo un estudio realizado por el Club de la Excelencia en la Sostenibilidad sobre factores que incluyen las empresas en sus Códigos éticos o de conducta, el $88 \%$ de las empresas encuestadas incluyen la transparencia32.

31.- Que la política de responsabilidad social corporativa incluya los principios o compromisos que la empresa asuma voluntariamente en su relación con los distintos grupos de interés e identifique al menos:

a) Los objetivos de la política de responsabilidad social corporativa y el desarrollo de instrumentos de apoyo.

b) La estrategia corporativa relacionada con la sostenibilidad, el medio ambiente y las cuestiones sociales.

c) Las prácticas concretas en cuestiones relacionadas con: accionistas, empleados, clientes, proveedores, cuestiones sociales, medio ambiente, diversidad, responsabilidad fiscal, respeto de los derechos humanos y prevención de conductas ilegales.

d) Los métodos o sistemas de seguimiento de los resultados de la aplicación de las prácticas concretas señaladas en la letra anterior, los riesgos asociados y su gestión.

e) Los mecanismos de supervisión del riesgo no financiero, la ética y la conducta empresarial.

32.- "Guía sobre Sistema de Gestión de la Ética en la Empresa", edita Club de la Excelencia en la Sostenibilidad" (página 34) 
Pero, aun siendo importante el valor de la transparencia en la gestión para mejorar la reputación de las empresas ante sus grupos de interés, todavía hay mucho camino por andar, y en ese recorrido hay que superar distintos espacios de gestión que aún siguen siendo zonas de información opaca, como son los relativos a la información de las retribuciones de consejeros y directivos de las empresas y a la información fiscal de empresas y particulares, y esto se sigue dando a pesar de los escándalos que se conocen por los medios de comunicación y las medidas aprobadas por los poderes públicos.

Las sorpresas que se están produciendo en estos años de crisis, en el conocimiento de las excesivas retribuciones de directivos de empresas que han entrado en graves crisis, y en particular de entidades financieras, con contratos blindados con altas indemnizaciones, o la utilización de paraísos fiscales para la evasión de impuestos de empresas y particulares ${ }^{33}$, reflejan la falta de información previa que tienen los distintos grupos de interés de las empresas y sus directivos, llegando a extremos que llaman la atención, como es el desconocimiento que tienen los propios socios 0 accionistas, que en definitiva legalmente son los propietarios de las mismas.

\section{6.- Conclusión}

La transparencia de las empresas y otro tipo de entidades es una necesidad cada vez más demandada por los distintos grupos de interés vinculados a las empresas. La transparencia es un valor fundamental en la gestión socialmente responsable de las empresas, que mejora significativamente la imagen reputacional de las empresas y favorece su relación con cada uno de sus grupos de interés. La transparencia es una parte básica del compromiso de la empresa por el buen gobierno corporativo. La transparencia de las empresas debe ser fomentada, pero también exigida por los poderes públicos como así lo requieren los distintos organismos internacionales con los que España ha suscrito acuerdos y participa en sus decisiones. Además, las Administraciones públicas deben llevar a cabo una gestión eficaz y transparente. 


\section{Bibliografía}

Normativa: Real Decreto Legislativo 1/2010, de 2 de julio, por el que se aprueba el texto refundido de la Ley de Sociedades de Capital, BOE núm. 161, de 3 de julio de 2010.

Libro: Diversos documentos y autores. Título: "Responsabilidad Social de las Empresas", editado por el Ministerio de Trabajo y Asuntos Sociales (año 2008), que contiene los documentos: Informe del Foro de Expertos en Responsabilidad Social de las Empresas; Informe sobre aspectos sociales y medioambientales relacionados con la Responsabilidad Social de las Empresas que incluye artículos de Manuel Escudero, Tomas Garcia Perdiguero, Alberto Andreu Pinillo, Marta de la Cuesta González, Josep María Lozano y Regina Rivera Pedreira; Documento sobre RSE del Dialogo Social y Real Decreto 221/2008, de 15 de febrero, por el que se crea y regula el Consejo Estatal de Responsabilidad Social de las Empresas.

Documento en la web: http://www.pactomundial.org/category/aprendizaje/10-principios/Diez Principios del Pacto Mundial de Naciones Unidas en la Red Pacto Mundial España.

Documento en la web: http://www.ilo.org/wcmsp5/groups/public/一-ed_emp/-emp_ent/documents/publication/wcms_124924.pdf sobre la "Declaración Tripartita de principios sobre empresas multinacionales y política social" de la OIT.

Documento en la web: http://www.oecd.org/daf/inv/mne/MNEguidelinesESPANOL.pdf Líneas Directrices de la OCDE para empresas multinacionales, revisión 2011.

Documento en la web: http://www.europarl.europa.eu/summits/lis1_es.htm sobre las "Conclusiones de la Presidencia de la Cumbre Europea de Lisboa de 23 y 24 de marzo de 2000".

Documento: Libro Verde sobre RSE de la Comisión Europea, bajo el título "Fomentar un marco europeo para la responsabilidad social de las empresas" (año 2001).

Documento: Comunicación de la Comisión Europea sobre RSE dirigida al Parlamento Europeo, al Consejo, al Comité Económico y Social Europeo y la Comité de las Regiones el 25 de octubre de 2011, bajo el título de "Estrategia renovada de la UE para 2011-2014 sobre la responsabilidad social de las empresas".

Documento: Directiva 2014/95/UE del Parlamento Europeo y del Consejo de 22 de octubre de 2014 por la que se modifica la Directiva 2013/34/UE en lo que respecta a la divulgación de información no financiera e información sobre diversidad por parte de determinadas grandes empresas y determinados grupos. 
Libro: "Informe del Congreso de los Diputados para potenciar y promover la responsabilidad social de las empresas" editado por el Ministerio de Trabajo y Asuntos Sociales (año 2007).

Normativa: "Ley 2/2011, de 4 de marzo, de Economía Sostenible" BOE núm. 55, de 5 de marzo de 2011.

Documento: "Estrategia Española sobre Responsabilidad Social de las Empresas", Ministerio de Empleo y Seguridad Social (2014), http://www.empleo.gob.es/es/sec_trabajo/EERSE_WEB.pdf

Documento: "Código de buen gobierno de las sociedades cotizadas", editado por la Comisión Nacional de Mercado de Valores (2015).

http://www.cnmv.es/DocPortal/Publicaciones/CodigoGov/Codigo_buen_gobierno.pdf

Documento: "Guía sobre Sistema de Gestión de la Ética en la Empresa", edita Club de la Excelencia en la Sostenibilidad. http://clubsostenibilidad.org/f_publicaciones/etica.pdf

Documento: "Obligaciones fiscales de las empresas y Responsabilidad Social Corporativa", Informe del Observatorio de Responsabilidad Social Corporativa de junio 2014. http://observatoriorsc.org/wpcontent/uploads/2014/07/RSC_y_obligaciones_fiscales_OBRSC.pdf 